\title{
Keynote Talk: Data by the People, for the People
}

\author{
Mor Naaman \\ Rutgers University School of Communication, Information and Library Studies
}

\begin{abstract}
What can we learn from social media and community-contributed collections of information on the web? The most salient attribute of social media is the creation of an environment that promotes user contributions in the form of authoring, curation, discussion and re-use of content. This activity generates large volumes of data, including some types of data that were not previously available. Even more importantly, design decisions in these applications can directly influence the users' motivations to participate, and hugely affect the resultant data. I will discuss the cycle of social media, and argue that a 'holistic' approach to social media systems, which includes design of applications and user research, can advance data mining and information retrieval systems.

Using Flickr as an example, I will describe a study in which we examine what motivates users to add tags and geotags to their photos. The new data enables extraction of meaningful (not to say semantic) information from the Flickr collection. We use the extracted information, for example, to produce summaries and visualizations of the Flickr collection, making the repository more accessible and easier to search, browse and understand as it scales. In the process, the user input helps alleviate previously intractable problems in multimedia content analysis.
\end{abstract}

